\title{
System dynamics mechanism of cross-regional collaborative dispatch of emergency supplies based on multi-agent game
}

\author{
Ying Qiu ${ }^{1} \cdot$ Meng Shi ${ }^{1} \cdot$ Xinna Zhao ${ }^{1} \cdot$ Yongping Jing $^{1}$
}

Received: 13 September 2020 / Accepted: 14 February 2021

(c) The Author(s) 2021

\begin{abstract}
The cross-regional coordinated dispatch of emergency supplies is a complex issue involving multiple topics, which covers multiple relationships and is affected by multiple variables. In the face of severe emergencies, relief supplies inside a specific area are far from meeting the explosive demand for emergency supplies. Besides, the supply of emergency materials and the disaster areas often have a spatial mismatch. Considering the attributes of externalities and public goods of emergency rescue, there are many obstacles for Local administration of emergency (LAE) and emergency logistics enterprises (ELE) spontaneously carrying out emergency supplies across regions. To solve this complexity problem, this research abstracts higher-level administration of emergency (HAE), LAE and ELE as the main stakeholders, with which a tripartite evolutionary game (ETG) model and a system dynamic (SD) model are constructed to analyze the dynamic mechanism of the complex system and to carry out the numerical simulation of the three-party game process. All the analyses and tests in this study have proved that the strong supervision of HAE has a decisive impact on the realization of cross-regional coordinated dispatch of emergency supplies, and the financial rewards and punishments imposed by HAE on other entities can accelerate or delay the achievement of the equilibrium strategy. However, when HAE chooses not to regulate, the cooperation willingness of LAE affects a lot that all the stakeholders will eventually reach equilibrium at $(1,1,1)$ only if LAE chooses to actively carry out cross-regional coordinated dispatch of emergency supplies.
\end{abstract}

Keywords Cross-regional collaborative dispatch $\cdot$ Emergency supplies $\cdot$ System dynamics model $\cdot$ Multi-agent evolutionary game

\section{Introduction}

In recent years, severe emergencies have occurred frequently on a global scale, posing a huge threat to human survival and development. According to the statistics of the International Emergency Disaster Database (EM-DAT), the number of serious emergencies worldwide from 1990 to 2020 exceeded 22,000 causing direct economic losses of trillions

Xinna Zhao

zhaoxinna@bipt.edu.cn

Ying Qiu

qiuying@bipt.edu.cn

Meng Shi

631275246@qq.com

Yongping Jing

jingyongping@bipt.edu.cn

1 School of Economics and Management, Beijing Institute of Petrochemical Technology, Beijing, China of dollars. Complexity, uncertainty and randomness are the basic characteristics of emergencies, which exacerbate the threat of security incidents to human life [1]. Taking into account emergencies' cross-border characteristics and the imbalanced rescue capacities of different regions, exploring a cross-regional coordinated responding mechanism for severer emergencies response has become a consensus. Cross-regional dispatch of emergency supplies is one of the core tasks in the process of constructing that responding mechanism, in which the dispatching mechanism, the evolution process of different stakeholders' strategic choices, and the multiple factors should be explored.

According to the existing experience, emergencies usually cause an explosive demand for materials instantly, and the quantity of this demand often exceeds the total amount of social inventory inside a specific region or even the entire society [2,3]. In China, after years of development, an emergency management system led by governments and extensively participated with enterprises and social forces 
has been formed. Undoubtedly, governments' regulation is divided into high-level supervision and local government supervision. Facing up with serious emergencies, rescue materials in a certain place or inside a specific region are often difficult to meet the explosive demand that crossregional coordinated dispatch of emergency supplies is necessary. Therefore, on one hand, local governments need to carry out emergency cross-regional allocations of materials, emergency procurement and even urgent production, and on the other hand the cooperation of emergency logistics enterprises is essential. However, there is often a spatial mismatch between the supply and demand of emergency materials that the spontaneous cross-regional dispatch of rescue supplies by local governments is held up. Will the regulation of HAE, such as the central government, helps realize the cross-regional dispatch of emergency supplies? How do HAE, LAE and ELE make strategic choices when implementing that cross-regional dispatch? What factors will affect those strategic choices?

In the research field of emergency supplies dispatching, researchers mainly focused on the decision-making problems or operational problems. For example, Alem et al. built a new two-stage random network model taking budget allocation, multi-vehicle fleet size, procurement issues and the impact of lead time into account to improve the efficiency of providing humanitarian assistance to the victims [4]. Fikar et al. constructed a simulation and optimization-based collaborative dispatch decision support system for emergency supplies based on mixed-integer programming, multi-agent simulation and other methods [5]. Li et al. comprehensively considering random demand and minimized transportation time, constructed a two-stage emergency material scheduling method based on Bayesian information update model [6].

Based on the above study, the operational problems of emergency supplies dispatch have been well studied. However, it is found that the complexity of cross-regional coordinated dispatch system is very high and the interactions of various variables on system stability also have strong uncertain and nonlinear characteristics. Therefore, it is not enough to have a comprehensive understanding for the complex relationships between stakeholders. Considering the complexity, the research paradigm of reductionism is adopted corresponding with the paradigm of the existing literature, which includes the research problems of decisionmaking of governments, games among multi-stakeholders, evolutionary dynamic mechanism of the complex system equilibrium, and so on [7]. Game theory has been widely used as an efficient methodology for decision-making [8] in the area of risk control and management $[9,10]$. Cooperation and conflict among different interested parties in crisis management are extensively investigated with game theory. Mcmillan et al. proposed a game-theoretic model to analyze
$R \& D$ performance differences in research-intensive companies sharing or hiding scientific information [11]. To explore the impact of asymmetry in terrorist's attributes between government and terrorist on defense equilibrium, Nikoofal and Zhuang built a game model that the government chooses between disclosure and secrecy of her defense system [12]. To well understand the quality problems in the construction market in China, Liu et al. proposed a principle-agent model to investigate construction project bidding and the root causes of the potential risks in the process of postearthquake reconstruction [13]. Besides, much more existing literature revealed the relationships among stakeholders in the research of different types of risk management, such as anti-terrorist $[14,15]$ and environmental risk control [16].

Nevertheless, most of the above research conducted with game theory assumes that all the stakeholders are completely rational, which neglects interested parties' bounded rationality in reality [17]. Therefore, researchers began to involve evolutionary game theory into the study of risk control and emergency management, which offered a feasible tool to achieve qualitative and quantitative analysis and to understand the evolutionary strategy choices by all the stakeholders and the exogenous influences, such as environmental pollution accidents $[18,19]$, and production accidents and disasters [20, 21].

Actually, EGT combines game theory with dynamic evolution theory based on the premise of limited rationality [22]. To perfect an emergency supplies dispatching system across regions and its associated management mechanism, it is necessary to understand the evolution mechanism and the complex behavioral decision-making among stakeholders systematic. Hence, with the use of system dynamics model, nonlinear behaviors abstracted out with EGT model can be simulated and discussed over time. The combined model with EGT and SD has been widely used in the research of risk control and emergency management [23-25] as well as logistics and supply chain management [26].

To sum up, although the many studies that have been conducted, some issues of the game relationships among different parties and the evolutionary decision-making process in emergency supplies dispatching are not clear. For example, how to define the game framework of the cross-regional emergency supplies dispatching? How to define the game interactions among HAE, LAE and ELE? Will the supervision and guidance of HAE, such as the central government, help realize the cross-regional dispatch of emergency supplies? How do HAE, LAE, and ELE make strategic choices when implementing cross-regional coordinated dispatch of emergency supplies? What factors will affect the strategic choices of the above entities? Therefore, in this study, the EGT is introduced to clarify the game relationship among various stakeholders in the collaborative dispatching process of emergency supplies across different regions, driven by 
which the complex and dynamic governance mechanism and also the policy of emergency supplying beyond the administrative boundary will be simulated and discussed with SD model. Hence, this study is an innovative attempt expanding the use of EGT-SD model to the researching area of emergency supplies dispatching and solving complex problems with non-liner and exogenous features in emergency logistics management, which is the major theoretical contribution.

The rest of the present paper is organized as follows. In the next section, we provide the theoretical model with EGT to analyze interactions among HAE, LAE and ELE in crossregional emergency supplies dispatching. In the following section, we propose EGT-SD model and present numerical simulation of the multi-agent evolutionary game. Finally, in the next section, we conclude important insights on crossregional collaborative dispatch of emergency supplies and put forward several worth learning suggestions.

\section{Evolutionary game analysis of interactions among HAE, LAE and ELE}

\section{Framework of cross-regional collaborative dispatch of emergency supplies}

Considering the externalities and the attributes of public goods of emergency rescue, market mechanisms cannot effectively achieve the coordinated dispatch of cross-regional emergency rescue supplies. Therefore, the cross-regional coordinated dispatch of relief supplies can only be carried out with the help of government regulation. However, the governments do not directly own materials and logistics resources that the cross-regional transfer of emergency supplies must be realized by ELE. Based on this, the complex system must cover the two types of governments and enterprises at the same time. Furthermore, China's emergency management system is a territorial management model with vertical constraints, which means that LAE is the direct responding subject and needs to be responsible for HAE. Therefore, this research divides government supervision into two major categories: high-level department supervision from HAE and local government guidance from LAE. The relationship between HAE, LAE and ELE is shown in Fig. 1. Based on existing research $[20,21]$ and the above analyses, there is a prominent contradiction between the crossregional coordinated dispatch of emergency resources and China's current territorial governance model of emergency management. Besides, it is suspectable that whether LAE makes coordinated strategic choices for emergency supplies dispatching considering the scarcity of resources. Therefore, the vertical supervision from HAE will affect the decisionmaking of LAE and ELE and is an extremely important power in the operation of the cross-regional coordinated dispatch of emergency supplies.

In conclusion, to obtain a better understanding for the operational mechanism of emergency supplies crossregional dispatch, this study abstracts HAE, LAE and ELE as the main stakeholders to construct an ETG model, and then build a SD model to carry out the numerical simulation of the three-party game process. The research process is illustrated as Fig. 2.

\section{Games among HAW, LAE and ELE}

In this study, the stakeholders of the tripartite evolutionary game are set as HAW, LAE and ELE, each of whom has two strategy choices. Therefore, there are eight sets of potential strategic combinations for the cross-regional dispatch of emergency supplies. HAE have two strategic choices, namely, strong supervision, incentives, and guidance to local governments, or no supervision of local governments. According to the above analysis, high-level government supervision will play an important role in the dynamic game of the system, and its strategic choice direction will become a reference for subsequent game scenario division. Local government departments have two strategic choices, namely, actively carrying out cross-regional coordinated dispatch of emergency materials and passively treating emergency materials. Finally, considering the profitability of the enterprise, emergency logistics companies also have

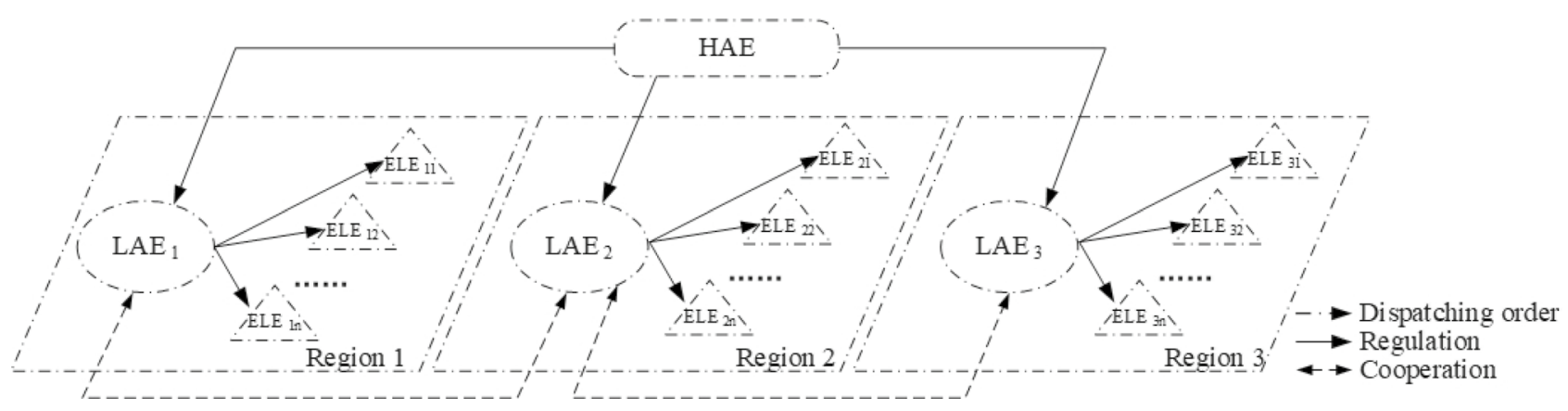

Fig. 1 Relationships among HAE, LAE and ELE 


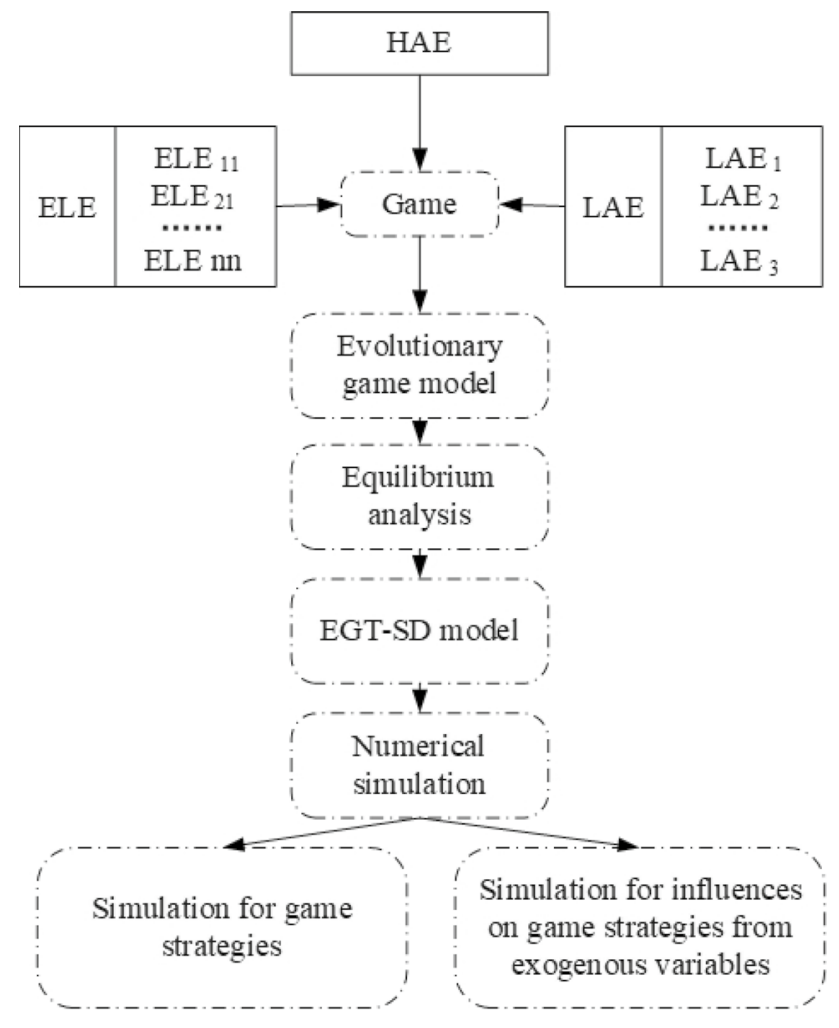

Fig. 2 Research process of modeling and simulating of this study

two strategic options, namely, cooperating with the local government's emergency logistics dispatching and violating the local government's emergency logistics dispatching. In the present research, $x, y$ and $z$ represent the probability of HAE choosing to regulate, local governments dispatching emergency supplies with collaborative willingness and logistics enterprises choosing to cooperate, respectively. Correspondingly, $1-x, 1-y, 1-z$ represent the possibility that HAE provides no regulation, that the local governments do not cooperate and that enterprises violate the local government's emergency supplies dispatch. The above-mentioned three-party game strategy will form eight potential strategy combinations, as shown in Fig. 2. All the tripartite game strategies are illustrated in Fig. 3.

\section{Parameters}

Based on the analysis of game relationships among multiagents, all the parameters involved in this study can be determined.

Parameters related to the intervention of HAE are defined as follows:

$M$ : compensation paid by HAE to LAE and ELE that independently initiate cross-regional supplies dispatch to emergency response.

$F$ : penalties imposed by HAE to LAE and ELE that passively respond to cross-regional supplies dispatch to emergency response.

A: rewards given by HAE to LAE and ELE for their collaborative cross-regional supplies dispatch to emergency response.

$P_{\mathrm{I}}$ : extra public benefits of HAE generated by the supervision of cross-regional coordinated dispatch for emergency supplies, such as the improvement of resource dispatching efficiency and the reduction of conflicts, etc.

$P_{G}$ : public benefits brought by the dispatch of emergency supplies, such as the satisfaction of rescue needs in a limited area, that are not affected by the intervention of HAE.

Parameters related to the cooperative dispatch of emergency supplies across regions by LAE and ELE are defined as follows:

$C_{\mathrm{a} 1}$ : costs occurred by LAE responding to independently supplies dispatch to emergency response.

$C_{\mathrm{a} 2}$ : costs occurred by ELE of autonomous dispatch of emergency supplies driven by market mechanisms and corporate responsibility.

$W_{\mathrm{a} 1}$ : losses suffered by LAE in emergencies.

$W_{\mathrm{a} 2}:$ losses suffered by ELE in emergencies.

$C_{\mathrm{t} 1}:$ cost paid for the cross-regional coordinated dispatch of emergency supplies by LAE proactively advocating the coordination.
Fig. 3 Strategic choices of stakeholders in tripartite games proposed in this paper

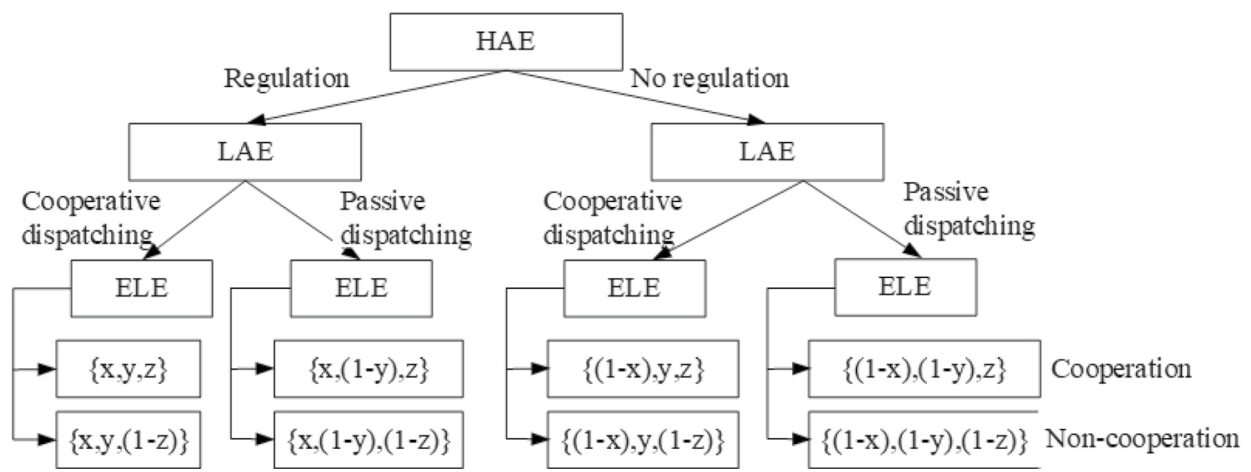


$C_{\mathrm{t} 2}:$ costs paid by the cross-regional coordinated dispatch of emergency supplies by ELE taking the initiative to implement the coordination.

$C_{\mathrm{t}}$ : costs paid for the cross-regional coordinated dispatch of emergency supplies when LAE and ELE jointly participate in the coordination.

$C_{\mathrm{R}}$ : regulatory cost of the HAE.

$C_{\mathrm{c}}$ : coordination cost of the HAE.

$P_{\text {a1 }}$ : benefits obtained by LAE dispatching emergency relief supplies to complete the emergency response.

$P_{\mathrm{a} 2}:$ profits obtained by ELE carrying out emergency rescue material dispatch to complete the emergency response.

$P_{t}$ : benefits obtained from the cross-regional coordinated dispatch of emergency supplies by LAE and ELE. $P_{\mathrm{t} 1}<P_{\mathrm{t}}$, $P_{\mathrm{t} 2}<P_{\mathrm{t}}$.

$\partial$ : external effects coefficient between LAE and ELE.

$\theta$ : influence level of the emergencies.

\section{Three-party evolutionary game model}

Eight behavioral strategy combinations of the three-party evolutionary game model will be developed, according to the behavioral choices and dynamic changes of HAE, LAE and ELE. Payoff matrix of EGT model could be construct from two opposing perspectives, which are regulated by governments or not regulated by governments. Therefore, in this paper, the payoff matrix is proposed from two views of regulating or not regulating by HAE, shown in Table 1, with all the profits and costs of all stakeholders.

Based on Table 1, the expected utility from the regulation of higher-level government is defined as:

$$
\begin{aligned}
U_{\mathrm{H} 1}= & y z\left[P_{\mathrm{I}}+P_{\mathrm{G}}+P_{\mathrm{t}}-C_{\mathrm{R}}-C_{\mathrm{C}}-2 A\right] \\
& +y(1-z)\left[P_{\mathrm{I}}+P_{\mathrm{G}}-C_{\mathrm{R}}-C_{\mathrm{C}}-M+F\right] \\
& +(1-y) z\left[P_{\mathrm{I}}+P_{\mathrm{G}}-C_{\mathrm{R}}-C_{\mathrm{C}}-M+F\right] \\
& +(1-y)(1-z)\left[P_{\mathrm{I}}+P_{\mathrm{G}}-C_{\mathrm{R}}-C_{\mathrm{C}}-2 F\right] .
\end{aligned}
$$

The expected utility of higher-level governments without regulation is defined as:

$$
\begin{aligned}
U_{\mathrm{H} 2}= & y z\left[P_{\mathrm{G}}-2 A\right]+y(1-z)\left[P_{\mathrm{G}}-M+F\right] \\
& +(1-y) z\left[P_{\mathrm{G}}-M+F\right]+(1-y)(1-z)\left[P_{\mathrm{G}}-2 F\right] .
\end{aligned}
$$

Correspondingly, the average expected return of HAE is:

$\overline{U_{\mathrm{H}}}=x U_{\mathrm{H} 1}+(1-x) U_{\mathrm{H} 2}$.

Similarly, the expected utility of lower-level government with collaborative willingness is defined as:

$$
\begin{aligned}
U_{\mathrm{L} 1} & =x z\left[P_{\mathrm{a} 1}+\partial P_{\mathrm{a} 2}+P_{\mathrm{t}}+A-C_{\mathrm{a} 1}-C_{\mathrm{t}}-(\partial+\theta) W_{\mathrm{a} 2}\right]+x(1-z)\left[P_{\mathrm{a} 1}+M-C_{\mathrm{a} 1}-C_{\mathrm{t} 1}-(\partial+\theta) W_{\mathrm{a} 2}\right] \\
& +(1-x) z\left[P_{\mathrm{a} 1}+\partial P_{\mathrm{a} 2}+P_{\mathrm{t}}-C_{\mathrm{a} 1}-C_{\mathrm{t}}-(\partial+\theta) W_{\mathrm{a} 2}\right]+(1-x)(1-z)\left[P_{\mathrm{a} 1}-C_{\mathrm{a} 1}-C_{\mathrm{t} 1}-(\partial+\theta) W_{\mathrm{a} 2}\right] .
\end{aligned}
$$

The expected utility of lower-level governments without collaborative willingness is defined as:

$$
\begin{aligned}
U_{\mathrm{L} 2} & =x z\left[P_{\mathrm{a} 1}+\partial P_{\mathrm{a} 2}-F-C_{\mathrm{a} 1}-\theta W_{\mathrm{a} 2}\right]+x(1-z)\left[P_{\mathrm{a} 1}-F-C_{\mathrm{a} 1}-\theta W_{\mathrm{a} 2}\right] \\
& +(1-x) z\left[P_{\mathrm{a} 1}+\partial P_{\mathrm{a} 2}-C_{\mathrm{a} 1}-\theta W_{\mathrm{a} 2}\right]+(1-x)(1-z)\left[P_{\mathrm{a} 1}-C_{\mathrm{a} 1}-\theta W_{\mathrm{a} 2}\right] .
\end{aligned}
$$

Table 1 The payoff matrix of the three-party evolutionary game model

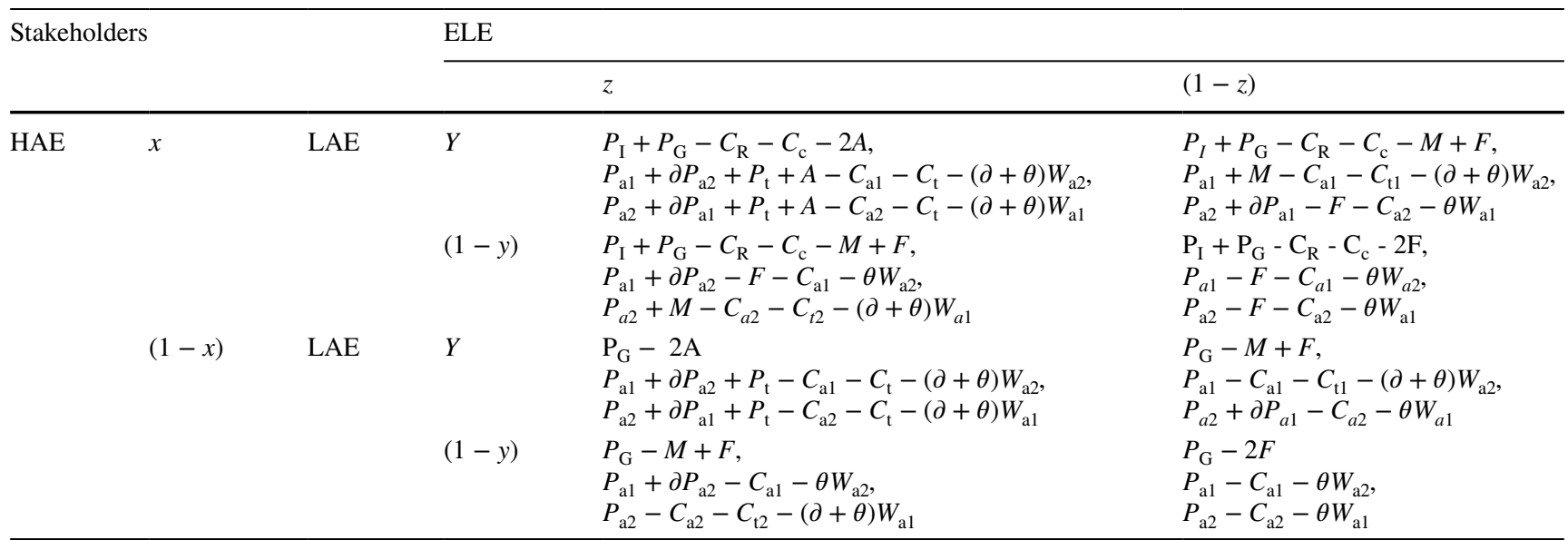


Correspondingly, the average expected return of LAE is:

$\overline{U_{\mathrm{L}}}=y U_{\mathrm{L} 1}+(1-y) U_{\mathrm{L} 2}$.

In the same way, the expected utility of ELE choosing to cooperate is defined as: find an analytical solution and it is much harder to judge the stability of the equilibrium solution by analyzing the eigenvalues of the Jacobian matrix.

To solve that complex problems, SD method is involved and the EGT-SD model is built. Based on feedback control theory and computer simulation technology, it can

$$
\begin{aligned}
U_{E 1} & =x y\left[P_{a 2}+\partial P_{a 1}+P_{t}+A-C_{a 2}-C_{t}-(\partial+\theta) W_{a 1}\right]+x(1-y)\left[P_{a 2}+M-C_{a 2}-C_{t 2}-(\partial+\theta) W_{a 1}\right] \\
& +(1-x) y\left[P_{a 2}+\partial P_{a 1}+P_{t}-C_{a 2}-C_{t}-(\partial+\theta) W_{a 1}\right]+(1-x)(1-y)\left[P_{a 2}-C_{a 2}-C_{t 2}-(\partial+\theta) W_{a 1}\right]
\end{aligned}
$$

The expected utility of ELE violating the local government's emergency supplies dispatch is defined as: effectively combine quantitative and qualitative analysis to establish a simulation platform, with which in-depth study

$$
\begin{aligned}
U_{\mathrm{E} 2} & =x y\left[P_{\mathrm{a} 2}+\partial P_{\mathrm{a} 1}-F-C_{\mathrm{a} 2}-\theta W_{a 1}\right]+x(1-y)\left[P_{\mathrm{a} 2}-F-C_{\mathrm{a} 2}-\theta W_{\mathrm{a} 1}\right] \\
& +(1-x) y\left[P_{\mathrm{a} 2}+\partial P_{\mathrm{a} 1}-C_{\mathrm{a} 2}-\theta W_{\mathrm{a} 1}\right]+(1-x)(1-y)\left[P_{\mathrm{a} 2}-C_{\mathrm{a} 2}-\theta W_{\mathrm{a} 1}\right] .
\end{aligned}
$$

Correspondingly, the average expected return of ELE is:

$\overline{U_{E}}=z U_{E 1}+(1-z) U_{E 2}$

After many rounds of trials and errors, and learning, the tripartite stakeholders adjust their strategies according to the outcomes of the game. Refer to the research of Friedman's, we construct the replicated dynamic equations for HAE, LAE and ELE as follows:

$\left\{\begin{array}{l}\frac{\mathrm{d} x}{\mathrm{~d} t}=x\left(U_{\mathrm{H} 1}-\overline{U_{\mathrm{H}}}\right) \\ \frac{\mathrm{d} y}{\mathrm{~d} t}=y\left(U_{\mathrm{L} 1}-\overline{U_{\mathrm{L}}}\right) \\ \frac{\mathrm{d} z}{\mathrm{~d} t}=z\left(U_{\mathrm{E} 1}-\overline{U_{\mathrm{E}}}\right)\end{array}\right.$

Substitute Eqs. (1)-(9) into (10), the evolutionary equations for HAE, LAE and ELE are obtained as follows: of information feedback behavior in complex systems can be well analyzed. To further discuss the co-evolution of HAE, LAE and ELE in the cross-regional coordinated dispatch of emergency supplies, and observe the trend of changes in various variables with the strategy of the three parties, this paper introduces multivariable, high-order and strong nonlinear SD model simulating the three-party evolutionary game. At the same time, it tries to change the relevant parameters to set different scenarios, observe the changes in the expected returns of each subject, and judge the most sensitive factors that affect the cross-regional coordinated dispatch of emergency supplies.

\section{Simulations of the multi-agent evolutionary} game

$\left\{\begin{array}{l}\frac{\mathrm{d} x}{\mathrm{~d} t}=x(1-x)\left(P_{\mathrm{I}}-C_{\mathrm{R}}-C_{\mathrm{C}}\right) \\ \frac{\mathrm{d} y}{\mathrm{~d} t}=y(1-y)\left[z\left(x A+P_{\mathrm{t}}\right)+x(1-z) M-\left(z C_{\mathrm{t}}+C_{\mathrm{t} 1}-z C_{\mathrm{t} 1}+\partial W_{\mathrm{a} 2}-x F\right)\right] \\ \frac{\mathrm{d} z}{\mathrm{~d} t}=z(1-z)\left[y\left(x A+P_{\mathrm{t}}\right)+x(1-y) M-\left(y C_{\mathrm{t}}+C_{\mathrm{t} 2}-y C_{\mathrm{t} 2}+\partial W_{\mathrm{a} 1}\right)\right] .\end{array}\right.$

The cross-regional coordinated dispatch system of emergency supplies is a typical complex system involving multiple subjects, in which the strategy selection of multiple subjects is affected by the combined effects of multiple factors in a dynamic and nonlinear way. By constructing an EGT mode, the dynamic equations of HAE, LAE and ELE are obtained, which are taken as the dynamic mechanism of the operation of the complex dispatching system of emergency supplies in fact. However, the above-mentioned replication dynamic equations involve the proportional distribution of the benefits of different strategy combinations impacted by many related parameters that it is difficult to

\section{SD-EGT model}

To further describe the dynamic decision-making process of the three stakeholders in EGT model, this study propose a SD-EGT model to describe the long-term evolution and trend of the decision-making process, simulated using Vensim ${ }^{\circledR}$ software. The constructing steps of SD-EGT model are as follows.

1. According to the payoff matrix and evolutionary equations of EGT model analyzed above, the main variables involved in SD-EGT model are: $M, F, A, P_{\mathrm{I}}, P_{\mathrm{G}}, C_{\mathrm{a} 1}, C_{\mathrm{a} 2}$, $W_{\mathrm{a} 1}, W_{\mathrm{a} 2}, C_{\mathrm{t} 1}, C_{\mathrm{t} 2}, C_{\mathrm{t}}, P_{\mathrm{a} 1}, P_{\mathrm{a} 2}, P_{t 1}, P_{\mathrm{t} 2}, P_{\mathrm{t}}, \partial$ and $\theta$. 


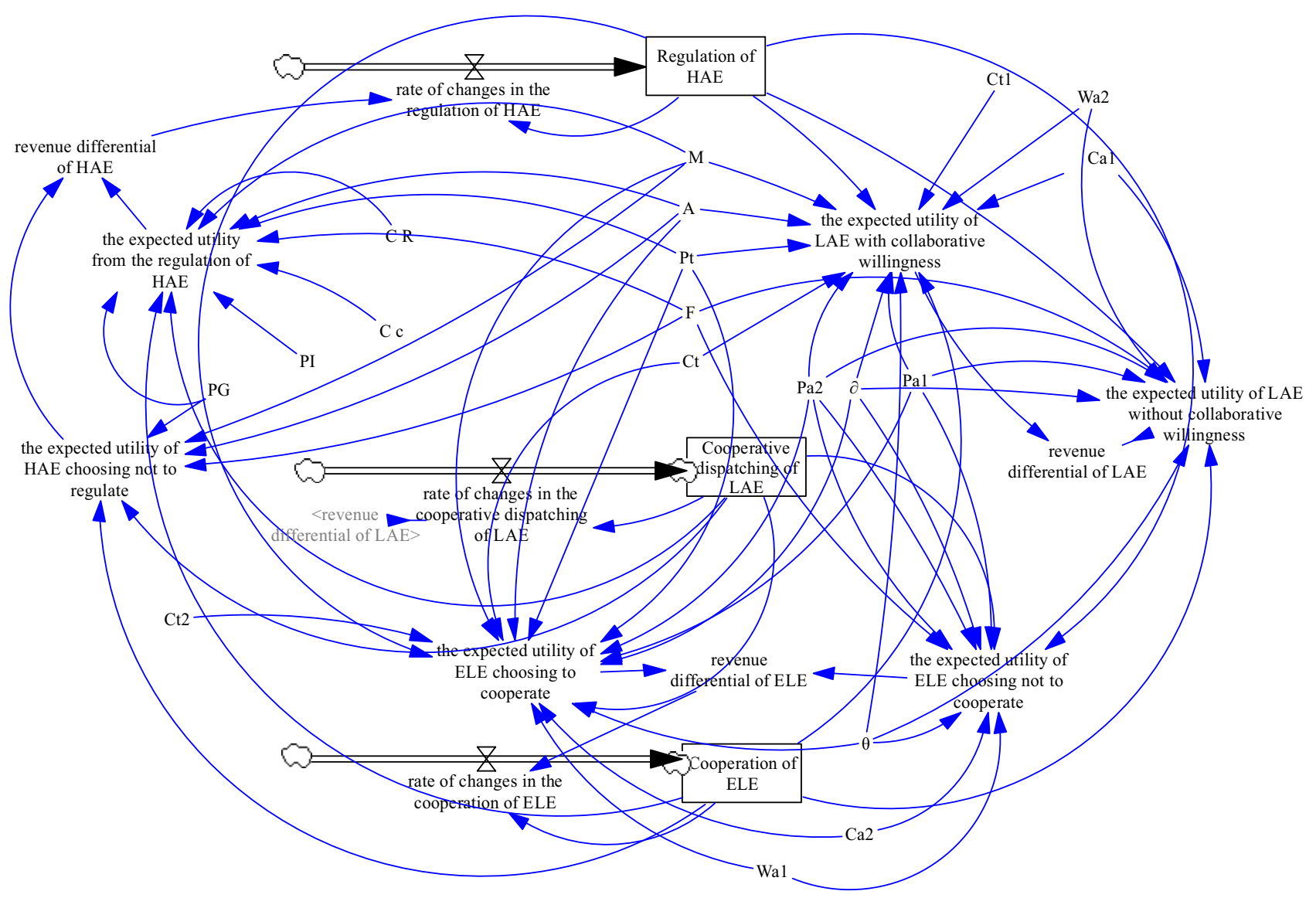

Fig. 4 System dynamic model based on the evolutionary game among HAE, LAE and ELE

The variables $U_{\mathrm{H} 1}$ and $U_{\mathrm{H} 2}$ represent the expected returns of regulation and non-regulation by $\mathrm{HAE}$, respectively. $U_{\mathrm{L} 1}$ and $U_{\mathrm{L} 2}$ represent the expected benefits of lower-level government with and without collaborative willingness, respectively. And $U_{\mathrm{E} 1}, U_{\mathrm{E} 2}$ are the representatives of the expected utilities of ELE choosing to cooperate with or violate the local government's emergency supplies dispatch.

2. The causal loop diagram of the built EGT model is given as Fig. 4. In this diagram, $x, y$ and $z$ are stock variables; $\frac{\mathrm{d} x}{\mathrm{~d} t}, \frac{\mathrm{d} y}{\mathrm{~d} t}$ and $\frac{\mathrm{d} z}{\mathrm{~d} t}$ represent three rate variables; $U_{\mathrm{H} 1}, U_{\mathrm{H} 2}$, $U_{\mathrm{L} 1}, U_{\mathrm{L} 2}, U_{\mathrm{E} 1}$ and $U_{\mathrm{E} 2}$ represent six intermediate variables; and $M, F, A, P_{\mathrm{I}}, P_{\mathrm{G}}, C_{\mathrm{a} 1}, C_{\mathrm{a} 2}, W_{\mathrm{a} 1}, W_{\mathrm{a} 2}, C_{\mathrm{t} 1}, C_{\mathrm{t} 2}, C_{\mathrm{t}}, C_{\mathrm{C}}, C_{\mathrm{R}}$, $P_{\mathrm{a} 1}, P_{\mathrm{a} 2}, P_{t 1}, \partial$ and $\theta$ are exogenous variables.

3. According to all the Eqs. (1)-(11) in the EGT model, the functional relationship of SD-EGT model among stock variables, rate variables and the exogenous variables are written.

In many cases, there is a lack of first-hand accurate information. The SD model reveals the behavior trend of the overall system and the influence of policy changes. The correctness of its model structure is more important than the accuracy of parameter values. It was also pointed out that the simulation model did not lie in how real the simulation process was, but focused on its usefulness and effectiveness and to what extent it revealed the law of changes in things and provided a decision-making reference for the evolution trend.

Therefore, the initial simulating data are set to satisfy the assumptions and analysis in Sect. 2 as follows:

$M=1, F=0.1, A=0.2, P_{\mathrm{I}}=0.3, P_{\mathrm{G}}=0.3, C_{\mathrm{a} 1}=0.2$, $C_{\mathrm{a} 2}=0.3, W_{\mathrm{a} 1}=0.3, W_{\mathrm{a} 2}=0.3, C_{\mathrm{t} 1}=0.2, C_{\mathrm{t} 2}=0.4, C_{\mathrm{t}}=0.3$, $C_{\mathrm{C}}=0.1, C_{\mathrm{R}}=0.1, P_{\mathrm{a} 1}=0.2, P_{\mathrm{a} 2}=0.2, P_{t 1}=0.5, \partial=0.4$ and $\theta=0.5$.

\section{Simulation analysis of pure game strategies}

It is clear that the complex system of cross-regional coordinated dispatch for emergency supplies will reach the evolutionary stable state through evolutionary analysis and stability analysis, while the evolutionary process of the system and the influencing paths of different variables are not determined. Therefore, the SD-ETG model is proposed and simulated with Vensim ${ }^{\circledR}$ software to further study the dynamic game relationships among the tripartite stakeholders.

Every agent of HAE, LAE and ELE has two strategy selections discussed in Section 2 that eight combinations, namely 
$(0,0,0),(0,1,0),(0,0,1),(0,1,1),(1,0,0),(1,1,0),(1,0,1)$ and $(1,1,1)$ are obtained. Based on the simulation results, pure strategy of the three stakeholders are hardly to change. However, the equilibrium states will be broken once any small change made by the any of the three agents. For example, we set the initial value as $(0.01,0.01,0.01)$ instead of $(0,0,0)$. Similarly, instead of $(1,1,1)$ as the initial value, $(0.99,0.99$, 0.99 ) could be used. In the present research, the simulation is conducted based on the two kinds of choices made by HAE. Namely, the initial value of the probability that HAE choose to regulate is set to 0.99 . Otherwise, the initial value of the probability HAE choosing not to regulate is set to 0.01 .

\section{Initial strategy of HAE is choosing to regulate}

According to the above analysis, there are four initial strategy combinations of $(1,1,1),(1,1,0),(1,0,1),(1,0,0)$ proposed by HAE, LAE and ELE when HAE chooses not to regulate. With a very small disturbance, such as 0.01 , set to every probability of strategy selection, it is confirmed that the gaming behaviors of the stakeholders in the tripartite evolutionary model have changed. Set the simulation cycle to 60 , i.e., INITIAL TIME as 0 , FINAL TIME as 60 , and

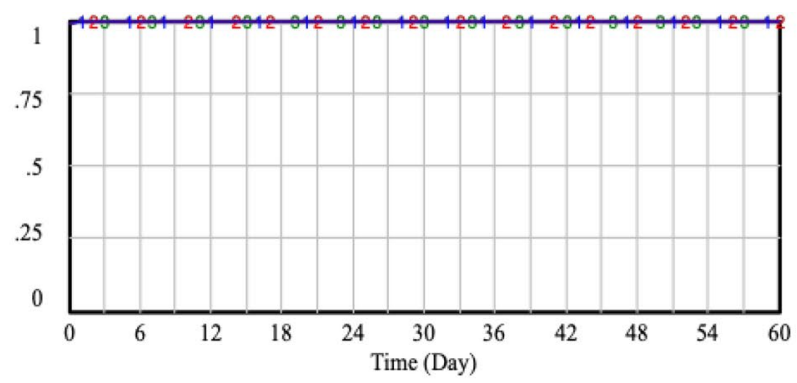

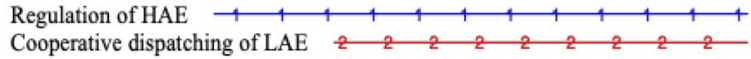

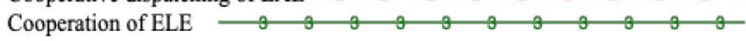

a Changes of stakeholders' gaming behaviors when their initial strategy combination is $(1,1,1)$

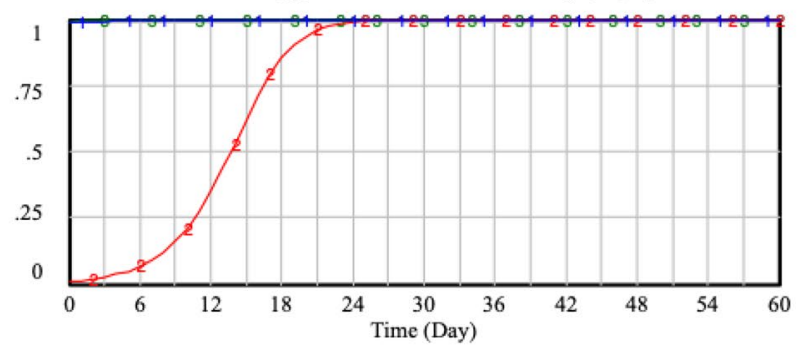

Regulation of $\mathrm{HAE}$

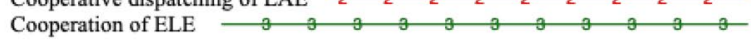

c Changes of stakeholders' gaming behaviors when their initial strategy combination is $(1,0,1)$
TIME STEP as 1 . The evolutionary process is illustrated in Fig. 5, in which the vertical coordinates represent the probabilities of stakeholders' strategy selections and the horizontal ordinate shows the simulated revolutionary time.

According to Fig. 5, regardless of the initial strategy of LAE and ELE, when HAE chooses to conduct strong supervision on the coordinated dispatch of emergency supplies across regions, the other two parties will eventually do the positive strategy selection through several times of trial and error, and learning. Finally, a positive strategic combination is made, that is, LAE will take the initiative to carry out cross-regional emergency material dispatch, and ELE will also take the initiative to give full play to the corporate social responsibility and actively cooperate with LAE's dispatch orders.

Therefore, under the high initial supervision intention of HAE, the three-party strategy will eventually converge to $(1,1,1)$, that is, HAE chooses strong supervision, LAE actively carries out cross-regional coordinated dispatch of emergency supplies, and ELE chooses to obey orders of LAE actively carrying out the cross-regional logistics business of emergency supplies.

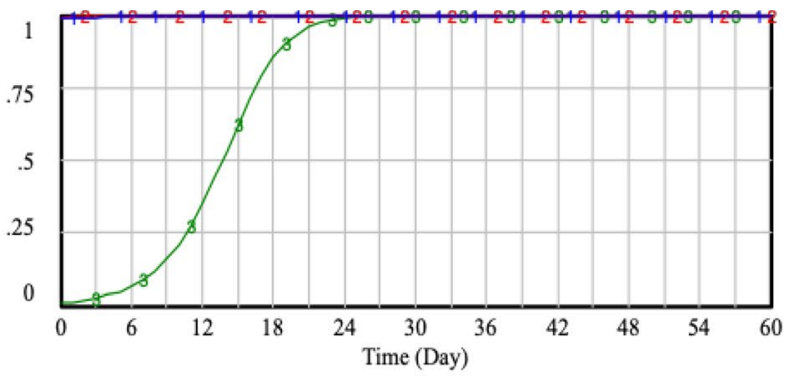

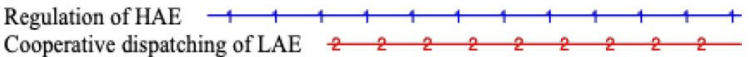
Cooperation of ELE

b Changes of stakeholders' gaming behaviors when their initial strategy combination is $(1,1,0)$

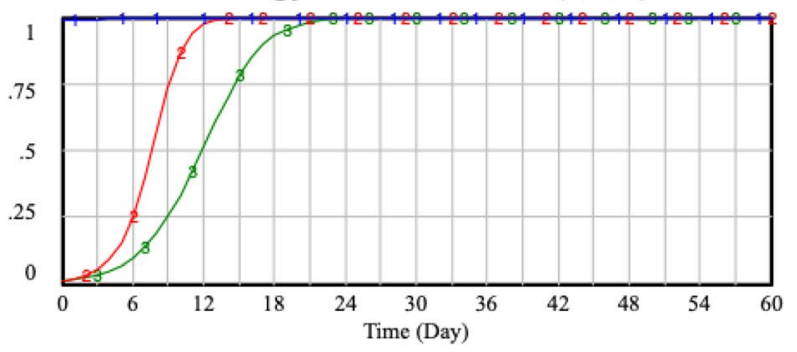

Regulation of $\mathrm{HAE}$

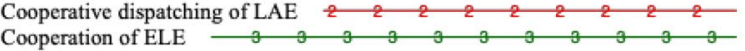

d Changes of stakeholders' gaming behaviors when their initial strategy combination is $(1,0,0)$

Fig. 5 Changes of stakeholders' gaming behaviors when HAE chooses to regulate 


\section{Initial strategy of HAE is choosing not to regulate}

When the HAE chooses not to supervise the coordinated dispatch of emergency supplies across regions, the three parties finally converged to two strategy choices $(1,1,1)$ and $(1,1,0)$ going through multiple strategic trial and error, and learning. The two choice combinations are that HAE will eventually passively supervise the cross-regional coordinated dispatch of emergency supplies, and LAE will only carry out crossregional emergency supplies dispatch through multiple attempts, but the strategic choice of ELE has greater uncertainty. Therefore, when HAE chooses not to supervise, the coordinated dispatch of emergency supplies across regions is difficult to achieve smoothly. Set the simulation cycle to 85, i.e., INITIAL TIME as 0, FINAL TIME as 85 , and TIME STEP as 1. The evolutionary process is illustrated in Fig. 6.

When HAE's willingness to supervise cross-regional emergency supplies is extremely low, the three entities will eventually reach the equilibrium at $(1,1,1)$ if LAE chooses to actively carry out the collaborative scheduling. However, if LAE chooses to passively carry out the coordinated dispatch, the three main bodies will eventually reach equilibrium at $(1,1,0)$ after repeated trial and error so that the regional coordinated emergency dispatch of rescue materials cannot be truly implemented.

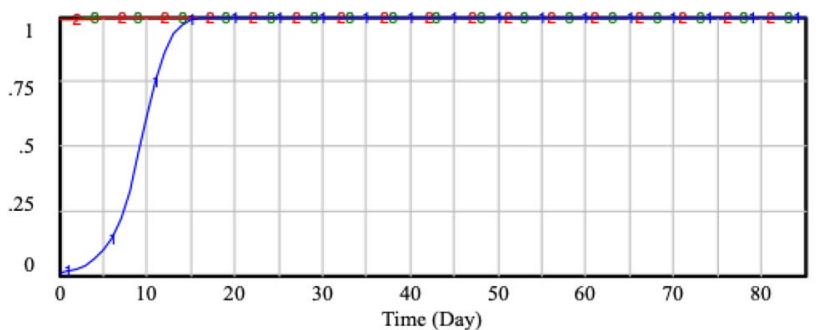

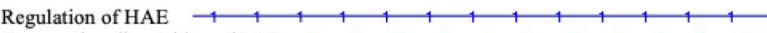
Cooperative dispatching of LAE $\begin{array}{llllllllllll}2 & 2 & 2 & 2 & 2 & 2 & 2 & 2 & 2 & 2 & 2\end{array}$

Cooperation of ELE

a Changes of stakeholders' gaming behaviors when their initial strategy combination is $(0,1,1)$

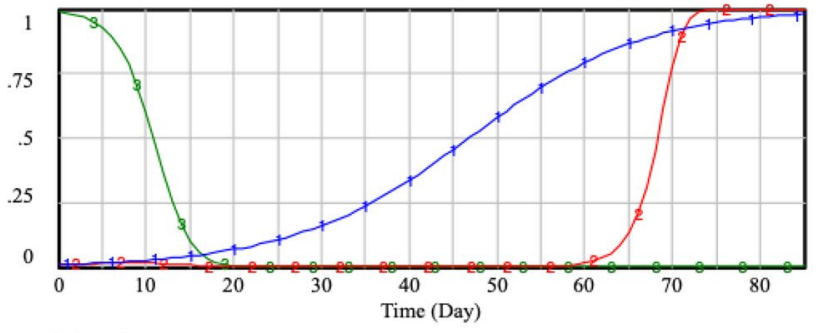

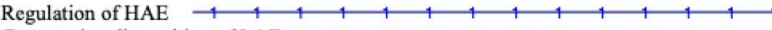

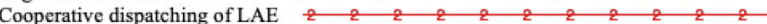
Cooperation of ELE

c Changes of stakeholders' gaming behaviors when their initial strategy combination is $(0,0,1)$

\section{Influences on game strategies from exogenous variables}

To further study the influences of some of the main exogenous variables on stakeholders' choices, simulations are conducted by changing the initial values of several key variables, where the evolutionary stability of $(1,0,0)$ is studied. In this part, it is mainly designed to find out whether the financial support or punishment from HAE impacts stakeholders' strategy selections.

This study believes that the vertical restraint of HAE has an important impact on the realization of cross-regional coordinated dispatch of emergency supplies. The previous analysis also confirms this view. According to the hypothesis of the article, the longitudinal constraints of HAE are mainly realized by three variables, namely $M, F$ and $A$. $M$ represents the subsidy given by HAE when LAE and ELE are actively and separately carrying out coordinated dispatch of emergency supplies. $F$ represents the punishment imposed by HAE when LAE and ELE negatively deal with the movement of materials across regions. $A$ represents the reward given by HAE when LAE and ELE take the initiative and cooperate in the coordinated dispatch of emergency supplies. Based on the analysis in Fig. 5, transform the assignments

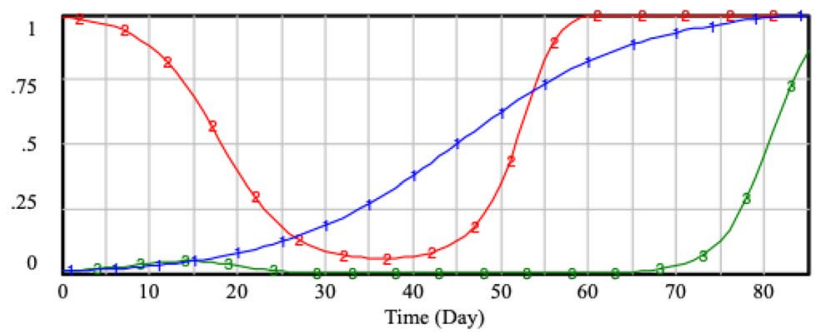

Regulation of $\mathrm{HAE}+1$ Cooperative dispatching of LAE $\begin{array}{lllllllllll}2 & 2 & 2 & 2 & 2 & 2 & 2 & 2 & 2 & 2 & 2\end{array}$ Cooperation of ELE

b Changes of stakeholders' gaming behaviors when their initial strategy combination is $(0,1,0)$

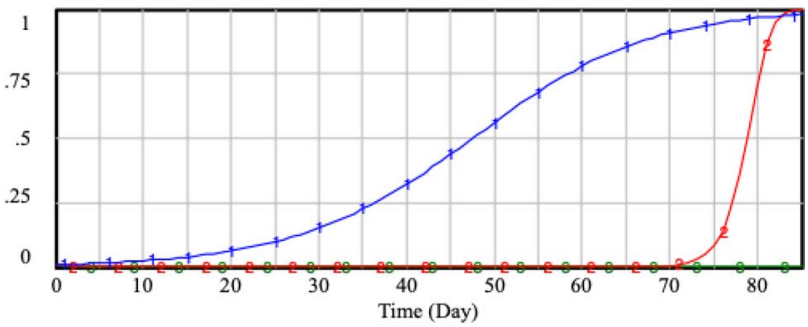

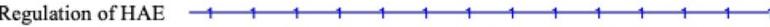
Cooperative dispatching of LAE $\begin{array}{llllllllllll}2 & 2 & 2 & 2 & 2 & 2 & 2 & 2 & 2 & 2 & 2\end{array}$

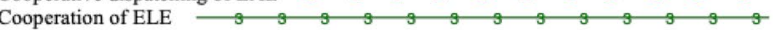

d Changes of stakeholders' gaming behaviors when their initial strategy combination is $(0,0,0)$

Fig. 6 Changes of stakeholders' gaming behaviors when HAE chooses not to regulate 
Table 2 Settings of simulation scenarios

\begin{tabular}{llll}
\hline Settings & $M$ & $F$ & $A$ \\
\hline Scenario 1 & 2 & 0.1 & 0.2 \\
& 0.3 & 0.1 & 0.2 \\
& 0.2 & 0.1 & 0.2 \\
Scenario 2 & 0 & 0.2 & 0.2 \\
& & 0.4 & 0.2 \\
& & 0.6 & 0.2 \\
& 1 & 0.2 & 0.2 \\
& & 0.4 & 0.2 \\
& & 0.6 & 0.2 \\
Scenario 3 & 0 & 0 & 0.6 or 2 \\
& 1 & 0.1 & 0.4 \\
& & & 0.6 \\
\hline
\end{tabular}

of $M, F$ and $A$, as shown in Table 2, to analyze the changes in the balance of stakeholder strategies.

In Fig. 5, the three parties of the game will converge to the $(1,1,1)$ strategy choice after 21 iterations if HAE has a strong willingness to monitor and LAE and ELE have a weak willingness to cooperate based on the initial value setting. That means cross-regional coordinated scheduling of emergency resources can soon be realized. Keep the initial choices of the above three parties and reduce or increase $M$, respectively. When $M=0.2$, the three parties of the game converge to the strategy choice of $(1,0,0)$ and cross-regional coordinated scheduling of emergency resources cannot be realized. The three parties finally converge to $(1,1,1)$ after more than 100 iterations with $M=0.3$, and the realization of cross-region coordinated resources scheduling is slow. However, the three parties only need about 10 iterations to converge to $(1,1,1)$ setting $M=2$, which means cross-regional coordinated materials dispatch across regions can be quickly realized (Fig. 7).

Beyond compensation, HAE can also impose penalties on stakeholders negatively responding to the coordinated resources dispatch across regions. Therefore, the value of $F$ is adjusted when $M=0$ and $M=1$ respectively in Scenario 2 , with which the influence only caused by $F$ and the superposition caused by $F$ and $M$ are analyzed.

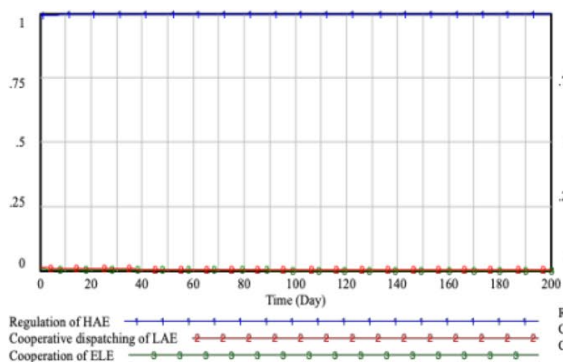

a $\mathrm{M}=0.2$

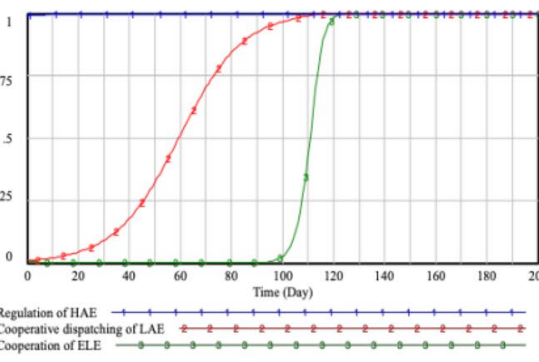

b $\mathrm{M}=0.3$

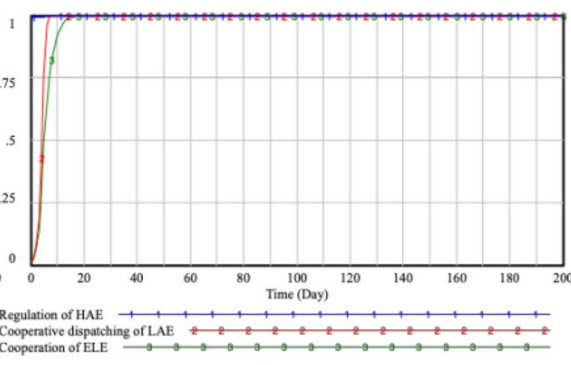

c $\mathrm{M}=2$

Fig. 7 Influences to strategy $(1,0,0)$ caused by $M$
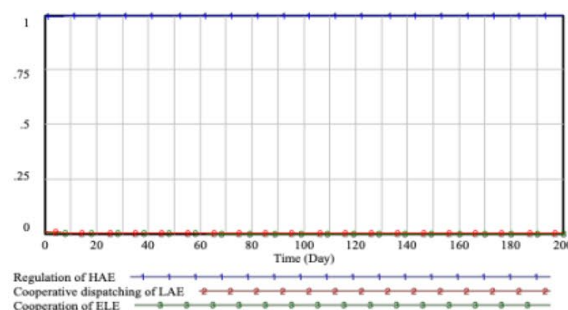

a $\mathrm{M}=0, \quad \mathrm{~F}=0.2$

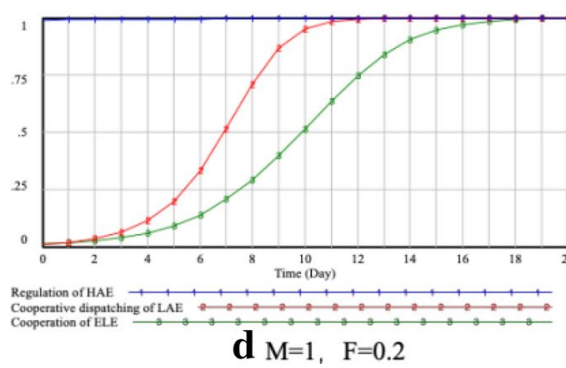

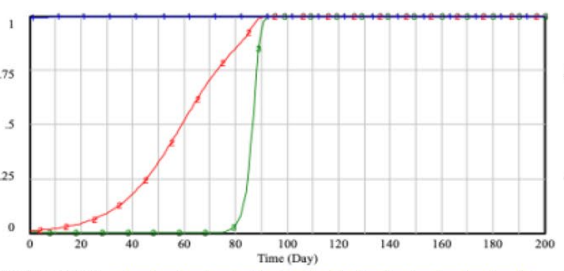

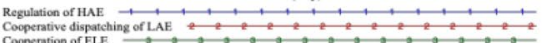

b $\mathrm{M}=0, \quad \mathrm{~F}=0.4$
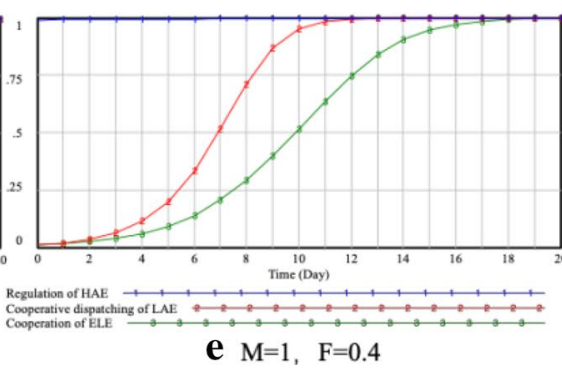
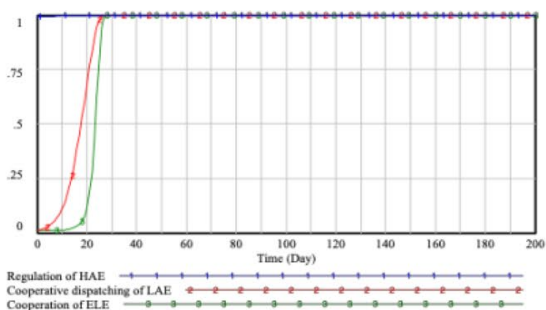

c $\mathrm{M}=0, \mathrm{~F}=0.6$

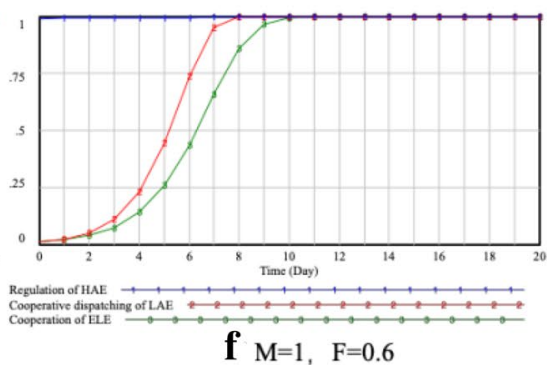

Fig. 8 Influences to strategy $(1,0,0)$ caused by $F$ and $M$ 
As shown in Fig. 8, when $M=0$, neither LAE nor ELE will actively cooperate in the regional coordination if the potential penalty imposed by HAE is low. With the intensification of HAE's potential punishment, LAE and ELE gradually form the cooperative willingness. The larger $F$ is, the faster the willingness is formed. When $M=1$, HAE not only grants subsidies to subjects who actively respond, but also imposes penalties on subjects who do not cooperate that the three parties can quickly reach a strategy combination of $(1,1,1)$.

To encourage LAE and ELE to actively increase the enthusiasm for participation, HAE provides rewards for spontaneous cooperation between the two parties, such as rewards for LAE and special financial support for enterprises. If HAE does not provide subsidies for LAE and ELE's coordination, nor imposes high potential punishment for non-cooperative behavior, it will not promote LAE and ELE to actively carry out emergency response no matter how much reward HAE grants to LAE and ELE. On the contrary, setting $M=1$ and $F=0.1$, the three parties converge to $(1,1,1)$ much faster as the reward increases. However, excessive rewards trigger a shock in the strategy selection process that elicits free-riding behaviors (Fig. 9).

\section{Conclusions}

In the face of sever emergencies, the emergency supplies inside a specific area are often difficult to meet the explosive rescue demand. More seriously, there are mismatches between the production areas of emergency materials and the disaster areas. Considering the attributes of externalities and public goods of emergency rescue, there are many obstacles for LAE and ELE spontaneously carrying out emergency supplies across regions that the vertical administrative regulation from HAE is very important. This research abstracts HAE, LAE and ELE as the main stakeholders, with which an ETG-based SD model is constructed to analyze and simulate the dynamic mechanism of emergency dispatch of rescue materials.
All the analyses and tests in this study have proved that the strong supervision of HAE has a decisive impact on the realization of cross-regional coordinated dispatch of emergency supplies, and the financial rewards and punishments imposed by HAE on other entities can accelerate or delay the achievement of the equilibrium strategy. However, when HAE chooses not to regulate, the cooperation willingness of LAE affects a lot that all the stakeholders will eventually reach equilibrium at $(1,1,1)$ only if LAE chooses to actively carry out cross-regional coordinated dispatch of emergency supplies.

Based on this research, it is possible to achieve a deeper understanding of the main game relationship in the coordinated dispatch system of emergency supplies across regions, which is beneficial to the further research of dispatching mechanism. According to all the observed results, supervision policies or regulations by HAE should be issued and executed to form a stronger impetus for the realization of cross-regional coordinated dispatch of emergency supplies. Supports and encouragements provided by HAE are also certainly in great need, with which LAE and ELE could be more cooperative voluntarily and efficiently. Hence, administrative suggestions or management advices for HAE are proposed as follows. From on hand, as a key force, HAE must provide adequate compensation, impose strong penalties, and provide appropriate rewards to ensure and accelerate the formation of the cross-regional emergency resource coordination dispatching system. From the other hand, HAE needs to strengthen the prevention of free-riding behavior and to reduce the communication cost and to optimize the coordination process.

However, this study also has certain flaws. For example, the proposed dispatch system is a typical complex giant system, which has a narrow coverage and lacks in universality with the consideration only for three agents. In addition, due to the lack of real data support, the article adopts the form of numerical simulation, which cannot accurately reflect the real situation of cross-regional coordinated dispatch of emergency resources. In future,

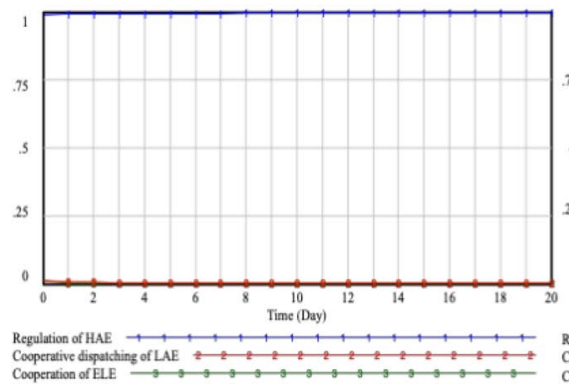

a $\mathrm{M}=0, \mathrm{~F}=0, \mathrm{~A}$ is an arbitrary value

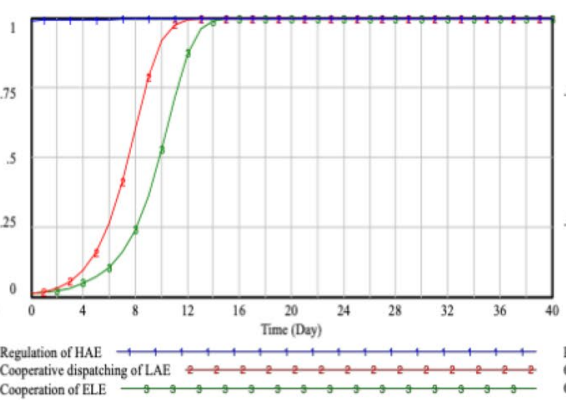

b $\mathrm{M}=1, \mathrm{~F}=0.1, \mathrm{~A}=0.6$

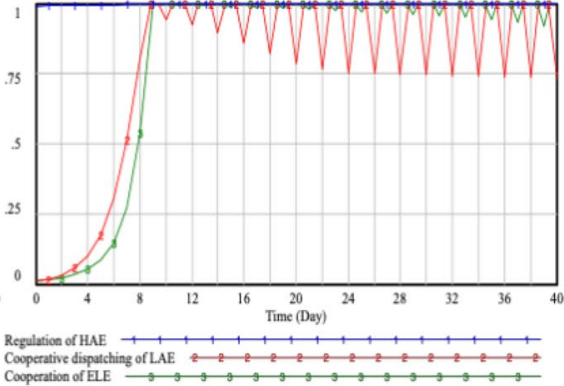

c $\mathrm{M}=1, \mathrm{~F}=0.1, \mathrm{~A}=2$

Fig. 9 Influences to strategy $(1,0,0)$ caused by $F, M$ and $A$ 
we will try to deepen the research for the complex relationships among more agents in the process of cross-regional resource dispatching for emergency logistics and strive to find more data support.

Acknowledgements This research was supported by Beijing Social Science Foundation [grant number 18GLB020 and 17JDGLB015].

Funding Beijing Social Science Foundation 18GLB020 and 17JDGLB015.

Availability of data and material The authors declare availability of data and material.

Code availability The authors declare code availability.

\section{Compliance with ethical standards}

Conflicts of interest/Competing interests On behalf of all authors, the corresponding author states that there is no conflict of interest.

Open Access This article is licensed under a Creative Commons Attribution 4.0 International License, which permits use, sharing, adaptation, distribution and reproduction in any medium or format, as long as you give appropriate credit to the original author(s) and the source, provide a link to the Creative Commons licence, and indicate if changes were made. The images or other third party material in this article are included in the article's Creative Commons licence, unless indicated otherwise in a credit line to the material. If material is not included in the article's Creative Commons licence and your intended use is not permitted by statutory regulation or exceeds the permitted use, you will need to obtain permission directly from the copyright holder. To view a copy of this licence, visit http://creativecommons.org/licenses/by/4.0/.

\section{References}

1. Tonmoy KB, Shakil A, Md SM (2018) Health, safety and quality management practices in construction sector: a case study. J Syst Manag Sci 8(2):47-64

2. Gong LJ, Zhang JH (2018) Research on construction and optimization's basic theory of emergency relief materials mobilization Chain. J Beijing Inst Technol (Social Sci Ed) 20(2):117-123

3. Heaslip G, Sharif AM, Althonayan A (2012) Employing a systems-based perspective to the identification of inter-relationships within humanitarian logistics. Int J Prod Econ 139(2):377-392

4. Alem D, Clark A, Moreno A (2016) Stochastic network models for logistics planning in disaster relief. Eur J Oper Res 255(1):187-206

5. Fikar, C., Gronalt, M. and Hirsch, P. A decision support system for coordinated disaster relief distribution. Expert Syst Appli $57: 104-116$

6. Li SL, Ma ZJ, Teo KL (2020) A new model for road network repair after natural disasters: Integrating logistics support scheduling with repair crew scheduling and routing activities. Comput Ind Eng 145:106506

7. Guo G, Cheng G (2019) Mathematical modelling and application for simulation of water pollution accidents. Process Saf Environ Prot 127:189-196

8. Toupo DFP, Strogatz SH, Cohen JD, Rand DG (2015) Evolutionary game dynamics of controlled and automatic decision-making. Chaos Interdiscipl J Nonlinearence 25(7):332-361
9. Yu JR, Liu XL, Zheng XD, Tao Y (2017) Selection intensity and risk-dominant strategy: A two-strategy stochastic evolutionary game dynamics in finite population. Appl Math Comput 297:1-7

10. Wang L, Zhao N, Liu DH (2020) Complex disaster management: a dynamic game among the government, enterprises, and residents. J Clean Prod 266:1-10

11. Mcmillan GS, Klavanst RA, Iii RDH (1995) Firm management of scientific information: some predictors and implications of openness versus secrecy. R\&D Manag 25(4):411-419

12. Nikoofal ME, Zhuang J (2015) On the value of exposure and secrecy of defense system: first-mover advantage vs. Robust Eur J Oper Res 246(1):320-330

13. Liu DH, Xu WJ, Li HY, Zhang WG, Wang WG (2011) Moral hazard and adverse selection in Chinese construction tender market: a case of Wenchuan earthquake. Disaster Prev Manag 20(4):363-377

14. Shan X, Zhuang J (2018) Modeling cumulative defensive resource allocation against a strategic attacker in a multi-period multi-target sequential game. Reliab Eng Syst Saf 179:12-26

15. Zhang J, Zhuang J (2019) Modeling a multi-target attackerdefender game with multiple attack types. Reliab Eng Syst Saf 185:465-475

16. Liu DH, Su Y (2014) Scenario optimal model of social mediation, information warning and police deploy in mass incident. Syst EngTheory \& Pract 34:2609-2618

17. Xu L, Zhou ZY, Du JG (2020) An evolutionary game model for the multi-agent co-governance of agricultural non-point source pollution control under intensive management pattern in China. Int J Environ Res Public Health 17(7):2472

18. Rocha ABS, Salomao GM (2019) Environmental policy regulation and corporate compliance in evolutionary game models with wellmixed and structured populations. Eur J Oper Res 279(2):486-501

19. Sun Z, Zhang W (2019) Do government regulations prevent greenwashing? An evolutionary game analysis of heterogeneous enterprises. J Clean Prod 231:1489-1502

20. Qiu Y, Shi XL, Cheng ZC (2018) Evolutionary game models on regional administrative collaborations to accidents and disasters. J Interdiscipl Math 21(4):807-823

21. Qiu Y, Shi XL, Hua GW (2019) Regional cooperative strategies for emergency response to accidents and disasters under longitudinal administrative constraint. Manag Rev 31(8):240-249

22. Long RY, Yang JH, Chen H, Li QW, Fang WQ, Wang L (2019) Co-evolutionary simulation study of multiple stakeholders in the take-out waste recycling industry chain. J Environ Manag 231:701-713

23. Liu QL, Li XC, Meng XF (2019) Effectiveness research on the multi-player evolutionary game of coal-mine safety regulation in China based on system dynamics. Saf Sci 111:224-233

24. You MJ, Li S, Li DW, Cao QR, Xu F (2020) Evolutionary game analysis of coal-mine enterprise internal safety inspection system in China based on system dynamics. Resour Policy 67:101673

25. Ma L, Liu QL, Qiu ZX, Peng YM (2020) Evolutionary game analysis of state inspection behaviour for coal enterprise safety based on system dynamics. SustainComput Inf Syst 28:100430

26. Tian YH, Govindan K, Zhu QH (2014) A system dynamics model based on evolutionary game theory for green supply chain management diffusion among Chinese manufacturers. J Clean Prod 80:96-105

Publisher's Note Springer Nature remains neutral with regard to jurisdictional claims in published maps and institutional affiliations. 